\title{
DESIGN OF HIGH-SPEED ROBOT LEG BASED ON DOUBLE FOUR-BAR MECHANISM
}

\author{
Zhang Yongnian ${ }^{1}$, Wu Yang $^{1}$, Xiao Maohua ${ }^{1}$, Wang Xiaochan ${ }^{1}$ \\ ${ }^{1}$ College of Engineering, Nanjing Agricultural University, Nanjing 210031, China \\ E-mail: wangxiaochan@njau.edu.cn
}

\begin{abstract}
High-speed ground motion is inevitably related to high acceleration and large loads on the leg. This imposes a challenge in robot leg design. This paper introduces an novel electric-drive robot leg based on double four-bar mechanism which is used to transform continuous rotation of motors to back and forth motion of the leg. This scheme avoids the ever-changing status of frequent acceleration and deceleration of the motors, and thus may reach higher rotation speed. Then the crank trajectory is optimized by inserting a piece of uniform motion, and the rocker trajectory is interpolated to obtain the optimized foot trajectory. These make the peak angular velocity decreased by $18.9 \%$ and the motion stability improved. In addition, the multiple lighten treatment using finite element analysis software is adopted to decrease leg's mass and rotary inertia under the premise of guaranteeing the safety of leg strength. Considering the fact that a tendon-bone colocation architecture not only provides compliance in the leg, but can also reduce bone stresses caused by bending on structures, we attach an elastic tendon to the ankle to reduce the pulse stress on the leg. Finally, the prototypes of robot leg are built to conduct the no-load walking experiment in the self-developed test bench of one-legged movement. The experiment results demonstrate that the highest walking speed can reach $1 \mathrm{~m} / \mathrm{s}$, which is 2.2 times leg length per second. Meanwhile, the peak torques of hip, elbow motor are markedly reduced by attaching the elastic tendon. These results verify the correctness and validity of the proposed design in this paper.
\end{abstract}

Keywords: High-Speed; Double Four-Bar Mechanism; Lighten Treatment; Elastic Tendon; Robot Leg.

\section{Introduction}

Quadruped robot is gradually transforming from the initial static walking gait into dynamic trot gait ${ }^{[1]}$, its locomotion speed is gradually increasing and is moving towards high-speed walking.

In nature, high-speed locomotion of animals is mainly achieved by high pace frequency and low duty cycle ${ }^{[2]}$. In the flight phase, legs fast forward and change the locomotion direction at the beginning and end, which requires high acceleration of legs. In the support phase, a great ground support force is required during high-speed locomotion. As a man is running at a speed of $4.5 \mathrm{~m} / \mathrm{s}$, his single leg support force is about 3 times of his weight ${ }^{[3]}$, and as a dog is galloping at a speed of $9 \mathrm{~m} / \mathrm{s}$, its single leg support force is about 2.6 times of its weight[4].

As a key component of quadruped robot, the freedom degree, layout form, self weight, etc. of leg mechanism have a great impact on the performance of robot locomotion ${ }^{[5]}$. To satisfy the high acceleration and large loads on the leg of robot during high-speed locomotion, the following methods are generally adopted in the design of the robot's leg:
(1) Increase the drive capability. The most typical example is the cheetah robot developed by the US MIT biomimetic robotics lab[6], the robot is capable of high-speed locomotion at a speed of $2.4 \mathrm{~m} / \mathrm{s}$ and straddling a $0.4 \mathrm{~m}$ high obstacle. Its superior performance is mainly accounted by the goodperformance and high-quality frameless and brushless DC torque motor (mass of $1 \mathrm{~kg}$, the peak torque of up to $60 \mathrm{Nm}^{[7]}$ ) independently developed by the laboratory is taken as the actuator, which can provide legs with high acceleration.

(2) Decrease locomotion inertia on legs. On the one hand, by placing all the drive motors in the body, the hip motor directly drives thigh locomotion, the knee motor transmits power to the lower leg via a transmission gear, which can effectively reduce rotary inertia on legs. For example, the knee joints of KOLT robot ${ }^{[8]}$ developed by Stanford University and Cheetah series robots ${ }^{[9]}$ developed by Swiss Federal Institute of Technology are all driven by ropes to reduce locomotion inertia on legs, the StarlETH robot ${ }^{[10]}$ developed by Swiss Federal University of Science and Technology drives the lower leg locomotion via chain drive; on the other hand, leg's mass is decreased as possible under the premise of 
meeting leg strength in leg design[11], thus decreasing locomotion inertia on legs.

(3) Add buffer devices. The BigDog developed by Boston Power Company[12], Cheetah robot ${ }^{[13]}$, the Cheetah robot developed by Swiss Federal Institute of Technology Zurich[9], and cheetah robot developed by MIT ${ }^{6]}$ all added springs or elastic tendons on legs as buffer devices.

In production of a robot prototype, (2) and (3) measures are easier to achieve, but in order to achieve a large acceleration of robot leg, selection of motors with larger power will lead to increase of leg weight, plus the high threshold of independent research of large torque motor technology and expensive cost.

Based on this, this paper introduces an novel electric-drive robot leg based on double four-bar mechanism which is used to transform continuous rotation of motors to back and forth motion of the leg. In this way, the forward and reverse motion of legs does not require forward and reverse rotation of motor, also avoiding the acceleration and deceleration requirements required there from, thereby effectively reducing locomotion cycle and achieving high-speed locomotion of quadruped robot.

\section{Overall Structural Scheme of Robot Leg}

To decrease rotary inertia on legs and improve the dynamic performance of the robot[14], on the one hand, we need to use lightweight materials and consider weight loss problems in design, on the other hand, we should try to arrange drive motor in the robot body. In this paper, the plane double fourbar mechanism shown in Fig. 1 is used to realize the locomotion of robot leg, in which $A$ and $D$ are fixed on the robot body, and are respectively fixed to the hip and knee motors, $A B C D$ constitute the crank and rocker mechanism, $D E F G$ constitute the doublerocker mechanism. $D H, H I, I K$ are respectively the thigh, lower leg and foot of the robot, the lower leg adopts a zoom parallel four-bar mechanism GHIJ to assure that thigh and foot are always in parallel, so as to increase leg stiffness.

In the robot leg structure shown in Fig.1, the locomotion of the robot thigh and the lower leg is respectively achieved by the following two four-bar mechanisms:

Crank and rocker mechanism $A B C D$ : the hip motor drives the crank $A B$ to conduct one-way whole-cycle rotation, and transmits locomotion to thigh rocker $C D$ through the connecting rod $B C$, thus achieving back and forth motion of rocker $C D$ as well as fast back and forth motion of the thigh $D H(D C H$ is a rigid body).

Double-rocker mechanism $D E F G$ : the knee motor drives back and forth motion of rocker $D E$, and transmits locomotion to rocker $G F(G F)$ is a rigid body) at knee joint through the connecting $\operatorname{rod} E F$, and fast up and down motion of the lower leg $\mathrm{HI}$ is achieved driven by the parallel four-bar mechanism GHIJ.

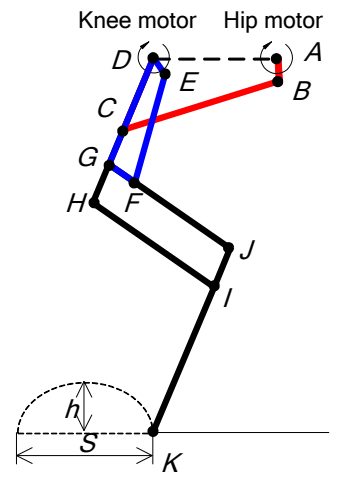

Figure 1: Designed robot leg

As for the tri-segmented leg of the robot shown in Fig.1, it has been shown that the femur ratio of the thigh, lower leg and foot in human and large quadruped animal legs is $0.39: 0.45: 0.16^{[15]}$, and the common femur ratio of small mammals is $0.33: 0.33: 0.33^{[16]}$, such configuration can bring them a larger working space and better acceleration performance. Therefore, this paper also uses the femur ratio, and the motion parameters and leg dimensions obtained via optimization algorithms are as shown in Tab.1:

Table1. Motion parameters and leg dimension $(\mathrm{mm})$

\begin{tabular}{|c|c|c|}
\hline Overall structure & Parameters & Length $/ \mathrm{mm}$ \\
\hline \multirow{3}{*}{$\begin{array}{c}\text { Motion } \\
\text { parameters }\end{array}$} & Step length $S$ & 300 \\
\hline & Step height $h$ & 70 \\
\hline & Cycle $T$ & $0.6 \mathrm{~s}$ \\
\hline \multirow{3}{*}{$\begin{array}{l}\text { Tri-segmented } \\
\text { leg }\end{array}$} & Thigh $L_{D H}$ & 150 \\
\hline & Lower leg & 150 \\
\hline & Foot $L_{I K}$ & 150 \\
\hline \multirow{4}{*}{$\begin{array}{c}\text { Crank and rocker } \\
\text { mechanism }\end{array}$} & Crank $L_{A B}$ & 22 \\
\hline & $\operatorname{rod} L_{B C}$ & 170.2 \\
\hline & Rocker $L_{C D}$ & 75 \\
\hline & Frame $L_{A D}$ & 130 \\
\hline \multirow{4}{*}{$\begin{array}{l}\text { Double-rocker } \\
\text { mechanism }\end{array}$} & Rocker $L_{D E}$ & 20 \\
\hline & $\operatorname{rod} L_{E F}$ & 105.5 \\
\hline & Rocker $L_{F G}$ & 32.5 \\
\hline & Frame $L_{D G}$ & 110 \\
\hline
\end{tabular}




\section{Optimization Trajectory \\ of Locomotion}

Foot K-point adopts a zero-impact foot trajectory developed by Beijing Institute of Technology[17], the angular velocity of rotation of the hip motor $(A B)$ and thigh $(D H)$ obtained via inverse kinematics is shown in Fig. 2. As can be seen from the figure, the angular velocity of hip motor is constantly less than 0 , namely the hip motor does not conduct forward and reverse switching along with the forward and backward motion of thigh, one-way rotation not only overcomes the inertia of back and forth locomotion[18], but also improves energy efficiency by using the rotation inertia.

However, the angular velocity of hip motor changes violently when $t=0.5 T=0.3 \mathrm{~s}$ (half of a locomotion cycle), it is easy to cause distortion of the system reaction and mutation of instantaneous acceleration of the motor. Therefore, a uniform rotation process is added in the locomotion of hip motor so that it meets: (1) to ensure the continuity of the two motors' angular velocity of rotation, and no mutations of acceleration; (2) to ensure the continuity of the foot trajectory, and the landing instantaneous velocity close to 0 .

In a locomotion cycle, the hip motor has four moments when acceleration is close to 0 , which are points $a, b, c, d$ in Fig. 2 . In order to ensure a smooth transition from the flight phase to the support phase during foot locomotion of the robot leg, hence $b c$ segment is improved to a constant velocity stage without changing the beginning and end; meanwhile, in order to ensure the continuity of crank locomotion, two points with a close speed are selected and the distance between two points is increased as much as possible to draw the angular velocity curves of the hip motor and thigh after improvement.

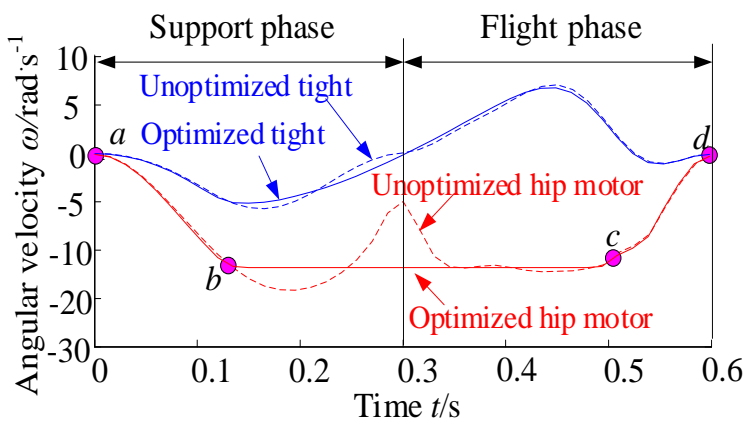

Figure 2: Angular velocity curves before and after improvement

As can be seen from the figure, the angular velocity of hip motor continuously changes after improvement, with no mutations of acceleration, the maximum angular velocity before improvement drops to 13.2079rad/s from 16.2891rad/s, a decrease of about $18.9 \%$.
In a quadruped robot one-legged mechanism, if the locomotion of knee joint remains unchanged, then the change of angular velocity of thigh will result in change of foot trajectory, this may have an impact on the robot locomotion, and need to re-plan the locomotion of knee joint.

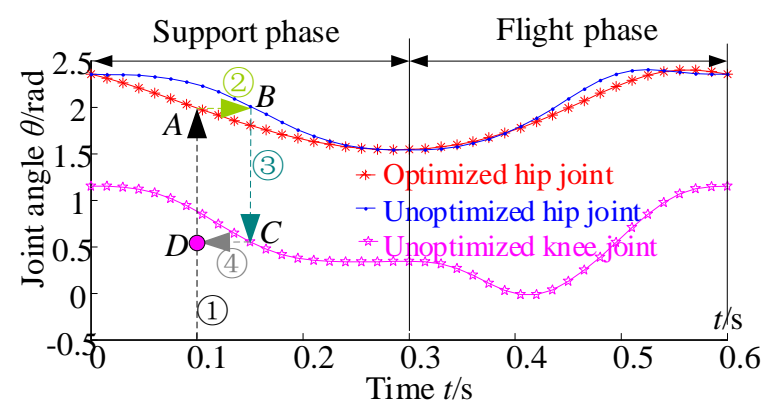

Figure 3: Flowchart of the knee angle solution

As shown in Fig. 3, to get the knee joint angle under a certain moment $t$, the thigh angle $A$ under this moment after improvement shall be obtained first, the moment $t^{\prime}$ corresponding to the same thigh angle $A$ before improvement is obtained via linear interpolation, namely to find point $B$ in figure, then obtain the knee joint angle corresponding to moment $t^{\prime}$, and take the angle as the improved knee joint angle at moment $t$, namely point $D$ in the figure.

Foot trajectory of hip and knee joint angle before and after improvement is shown in Fig. 4, as can be seen from the figure, the foot trajectory is basically identical before and after improvement, thus ensuring the smoothness of locomotion.

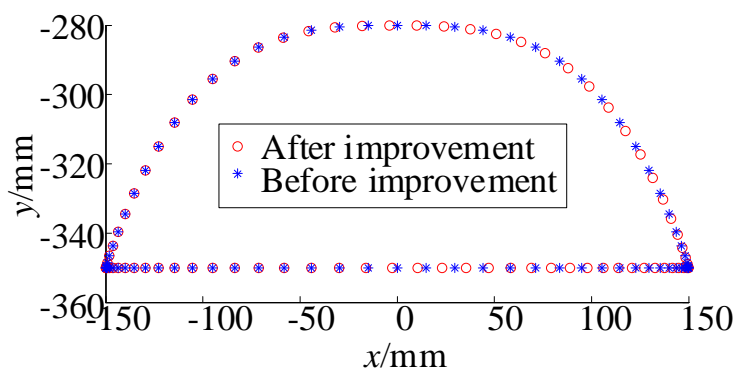

Figure 4: Foot trajectory before and after improvement

\section{Lighten Treatment}

In order to further improve the locomotion speed of robot leg, lighten treatment is conducted on the entire leg to make it meet the lightweight requirements of leg design, reduce locomotion inertia on legs and improve leg locomotion speed. Foot is the most important part of a tri-segmented leg, it is supported by the ground reaction force and it supports the entire leg. If the material reduction is unreasonable, it will result in insufficient mechanical strength of the leg, easily leading to fracture, optimization design of the foot is elaborated in the following part. 


\subsection{Theoretical calculation}

High-strength 6061 aluminum alloy is selected as the leg processing material, relevant mechanical manuals are referred to, the parameters shown in Table 2 are taken as the basis:

Table 2. Calculation parameters

\begin{tabular}{|l|l|}
\hline Parameters & Values \\
\hline Foot length $L / \mathrm{mm}$ & 150 \\
\hline Total mass $M / \mathrm{kg}$ & 20 \\
\hline Floor angle $\alpha{ }^{\circ}$ & 45.25 \\
\hline Walking coefficient $\eta$ & 1.5 \\
\hline Foot width-thickness ratio $h / b$ & 2 \\
\hline Yield strength $\sigma_{\mathrm{s}} / \mathrm{MPa}$ & 276 \\
\hline Safety coefficient $\eta_{b}$ & 5 \\
\hline
\end{tabular}

We can see from the calculation formula of bending strength:

$$
\sigma_{\max }=\frac{M_{\max }}{I_{z}} y_{\max } \leq[\sigma]
$$

Where, $M_{\max }=\eta M g \sin \alpha L, I_{z}=b h^{3} / 12, y_{\max }=h / 2$, $[\sigma]=\sigma_{s} / n_{b}$.

The calculated foot thickness is approximately $b \approx 9.541 \mathrm{~mm}$. In order to facilitate materials processing, foot thickness is rounded, and the thickness and width of the foot are obtained to be $b=10 \mathrm{~mm}, h=20 \mathrm{~mm}$.

\subsection{Selection of cross section}

Denote $W_{z}$ as the section modulus in bending, and $A$ as the cross section area, generally $W_{z} / A$ can be adopted to measure the rationality and economical efficiency of the shape of cross section.

The larger the specific value of $W_{z} / A$, the more economical and reasonable of the shape of cross section. Here we use an I-shaped cross section with a larger $W_{z} / A$.

As shown in Fig. 5, the reserved area is the Ishaped cross section after removing the materials with red dotted portions, and the design variables meet $0 \leq d \leq h / 2,0 \leq m \leq b$.

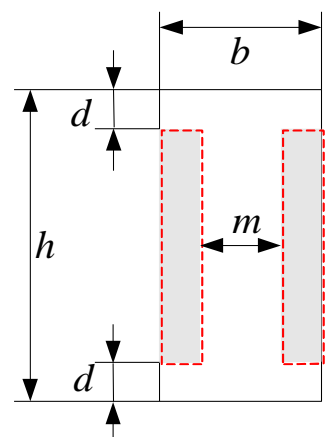

Figure 5: Shape of cross section

In MATLAB, fmincon function is invoked to find the maximum specific value of $W_{z} / A$ as target and to get the optimal $d$ and $m$, as shown in Fig. 6 .

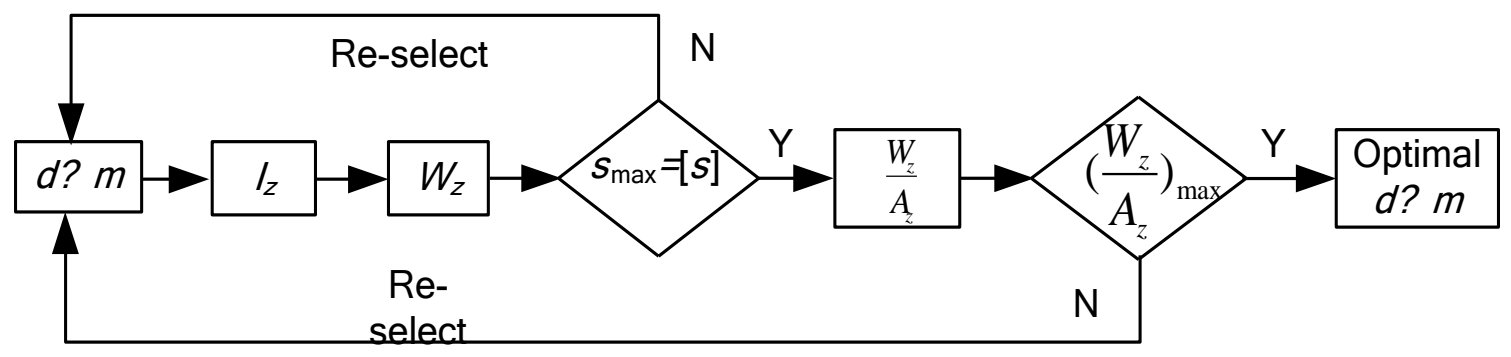

Figure 6: Block diagram of section optimization

The optimal $d=4 \mathrm{~mm}$ and $m=6 \mathrm{~mm}$ are obtained, thus obtaining the 3D model of the foot after the 1 st lighten treatment, namely, as shown in Fig. 7.

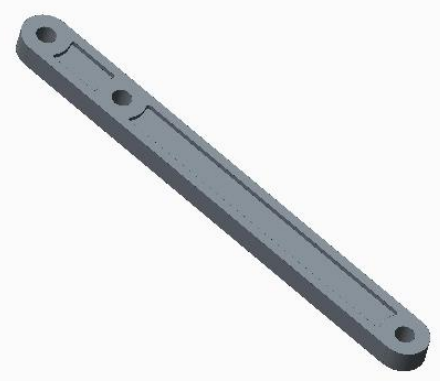

Figure 7: Foot model after 1st lightened treatment

\subsection{ANSYS topology optimization}

The goal of topology optimization is to find the optimal material distribution scheme for single-load or multi-load objects, reduce structural deformation and increase structural stiffness under the constraints of structure.

Unlike traditional optimization designs, topology optimization[19] does not require the definition of parameters and optimization variables. The objective function, state variables and design variables are pre-defined, and the user only needs to give the structural parameters (material properties, models, loads, etc.) and the percentage of material to be omitted. 
A foot model is created via Creo2.0 and based on the initial structure parameters, the foot model is led in Workbench14.0 for a finite element analysis through the interface between Creo2.0 and ANSYS, parameters shown in Tab. 3 are set in the system:

Table 3. Parameters of topology optimization

\begin{tabular}{|l|l|}
\hline Parameters & Definition \\
\hline Material properties & Aluminum Alloy \\
\hline Mesh size/mm & 1 \\
\hline Constraint type & Fixed Support \\
\hline Load type & Bearing Load \\
\hline $\begin{array}{l}\text { Percentage of material } \\
\text { removed }\end{array}$ & $30 \%$ \\
\hline
\end{tabular}

The results of finite element analysis are shown in Fig. 8, where gray part is where the materials are reserved and orange part is where the materials are removed.

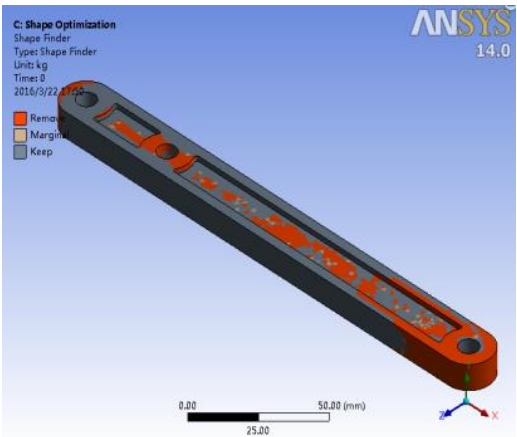

Figure 8: Optimization results of Workbench

After a second modification, the 3D model of the foot after 2nd lightened treatment is obtained, as shown in Fig. 9.

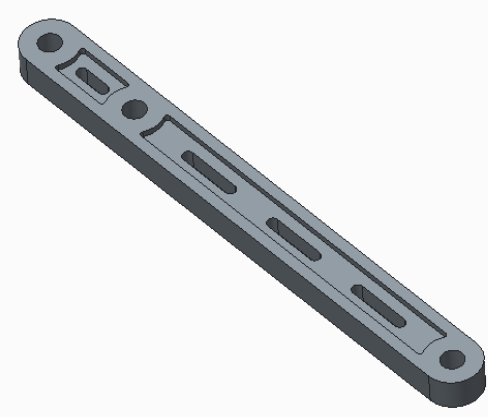

Figure 9: Foot model after 2nd lightened treatment

\subsection{Comparison analysis}

Fig. 10 shows the section nephogram of unlightened treatment, equivalent stress and total deformation nephogram of the 1st lighten treatment and 2nd lighten treatment with the same cross section.

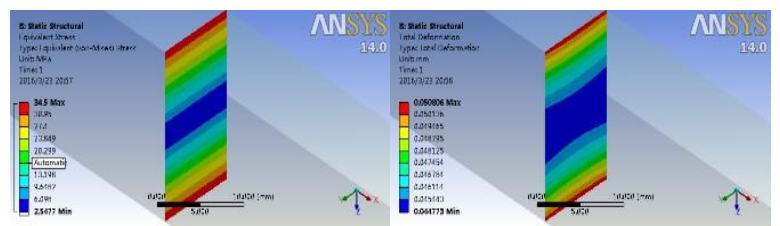

(a) Section nephogram of un-lightened treatment

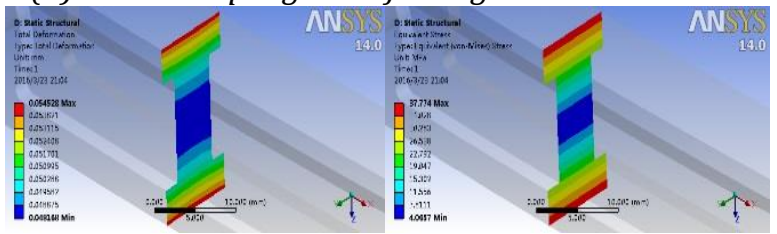

(b) Section nephogram after 1st lightened treatment

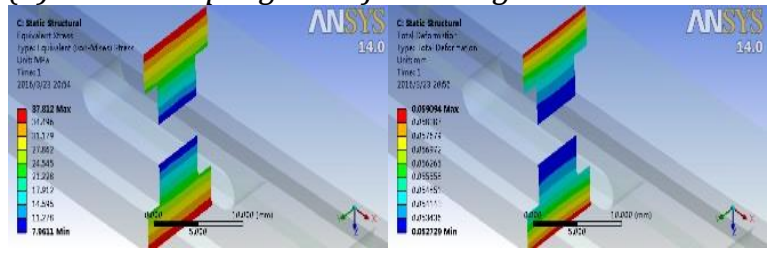

(c) Section nephogram after 2nd lightened treatment Figure 10: Comparison analysis of section nephogram

After the above two steps of lighten treatment, the foot mass decreased from $114.9 \mathrm{~g}$ to $88.0 \mathrm{~g}$, a decrease of $23.4 \%$, the $W_{z} / A$ of foot cross section increased from 3.333 to 4.007 , an increase of $20.2 \%$, proving the shape of cross section is more economical and reasonable. Although the equivalent stress and the cross section deformation of the same cross section of the foot are increased (as shown in Tab. 4), the increase is still within the permissible range and is reasonable, so this lighten treatment for the foot is correct and effective.

Table 4. Comparison before and after lightened

\begin{tabular}{|l|c|c|c|}
\hline & $\begin{array}{c}\text { Before } \\
\text { optimization }\end{array}$ & $\begin{array}{c}\text { After } \\
\text { optimization }\end{array}$ & Percentage \\
\hline Mass (g) & 114.9 & 88.0 & $-23.4 \%$ \\
\hline$W z / A$ & 3.333 & 4.007 & $+20.2 \%$ \\
\hline $\begin{array}{l}\text { Stress } \\
\text { (MPa) }\end{array}$ & 34.5 & 37.8 & $+9.6 \%$ \\
\hline $\begin{array}{l}\text { deformation } \\
(\mathrm{mm})\end{array}$ & 0.051 & 0.059 & $+15.7 \%$ \\
\hline
\end{tabular}

\section{Elastic Tendon Design}

Some studies ${ }^{[20,21]}$ have shown that during the locomotion of mammals, the ground impact force on animals is not only borne by the bones, but also by the elastic structures of the muscles, ligaments and other elastic structures (as shown in Fig. 11), which can withstand the load of great strength, play the role of energy storage and shock absorption, thus avoiding the direct stiff impact of the ground on legs, and improving the mechanical properties of the legs to a certain extent. 


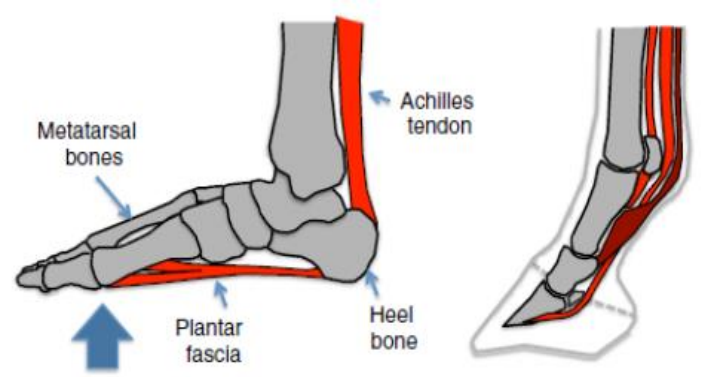

Figure 11: Human and horse foot

A passive elastic tendon is added between the heel and foot of the robot to improve the force structure of the leg in reference to the ligament principle of animals. As shown in Fig. 12, a simplified foot and ligament mechanism is added to the ankle joint $A$, one end of the elastic tendon is connected to $O$ on tiptoe, bypassing planta fascia $E$, through the foot, and the other end is connected to the parallel four-bar connection $C$ near the knee joint, and is fixed to $D$ on the triangular plate through a triangular plate that is connected by pins.

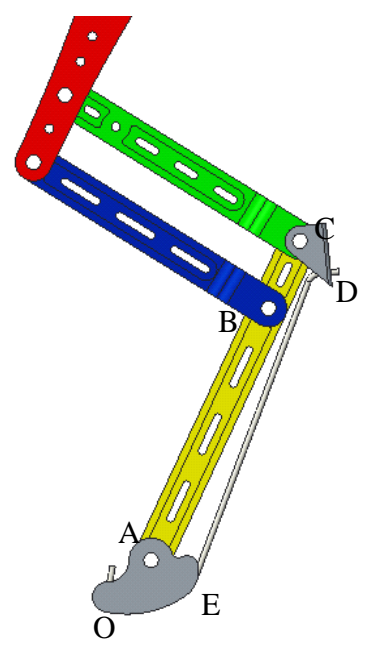

Figure 12: Designed robot leg with ligament

\subsection{Theoretical analysis}

During high-speed locomotion, the robot leg is subjected to a very large ground reactive force at the moment of landing. As shown in Fig. 13, it can be seen from the stress analysis that the planta fascia and elastic tendon form a triangle when robot leg touches the ground after adding an elastic tendon.

Compared to the single mechanical force on robot leg without the elastic tendon, part of the impact force is borne by the elastic tendon, thus relieving the ground impact effect on the robot leg, playing a role of shock absorption, preventing foot fracture, etc., but also storing energy, unleashing energy when robot leg transforms from the support phase to the flight phase, while achieving the flexible reversing locomotion of the legs.

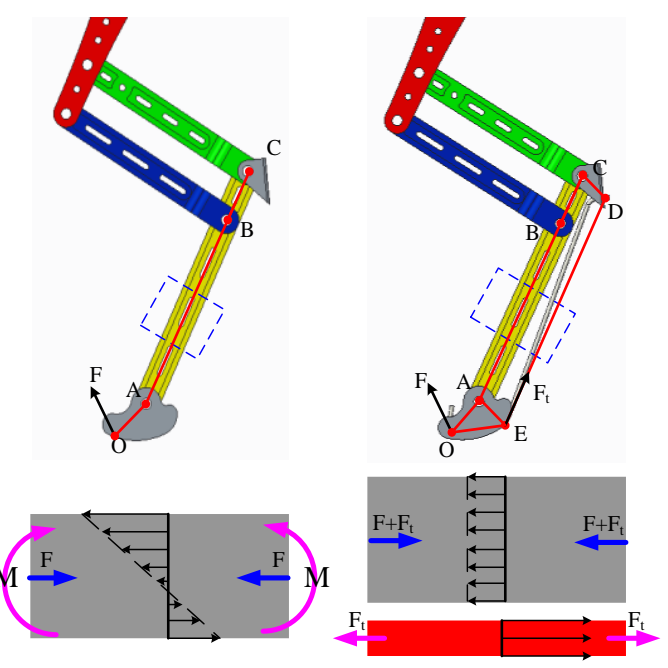

Figure 13: Conceptual stress profile on the foot

\subsection{ANSYS static analysis}

Set up a 3D model of the robot leg by Creo2.0 and change the position of leg mechanism so as to meet the above-mentioned initial angle at the moment of landing ground, thus facilitating comparison of theoretical analysis.

Import into Workbench14.0 for a finite element analysis, set the initial parameters, select the static analysis system, attribute definitions are as shown in Tab. 5.

Table 5. Parameters of static analysis

\begin{tabular}{|l|l|}
\hline Static analysis system & definition \\
\hline $\begin{array}{l}\text { Material properties } \\
\text { (elastic tendon) }\end{array}$ & Nylon \\
\hline $\begin{array}{l}\text { Material properties } \\
\text { (other) }\end{array}$ & $\begin{array}{l}\text { Aluminum } \\
\text { alloy }\end{array}$ \\
\hline Parts connection & Bonded \\
\hline Mesh size & 5mm \\
\hline Constraint type & $\begin{array}{l}\text { Fixed } \\
\text { Sunnort }\end{array}$ \\
\hline Load type & Force \\
\hline
\end{tabular}

Then conduct force loading and fixed constraints, load the same external force as the ground reaction force and direction at the toes, add fixed constraints on the thigh hole surface, set the mesh partition properties, process after adding the results, and obtain the finite element analysis results through operational analysis. Fig. 14 shows the strength analysis with and without the tendon, stress distribution of foot, deformation distribution of foot, and equivalent stress distribution of foot. 

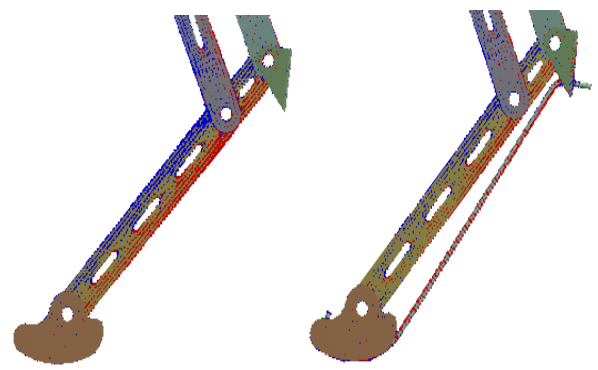

(a) Stress distribution of foot

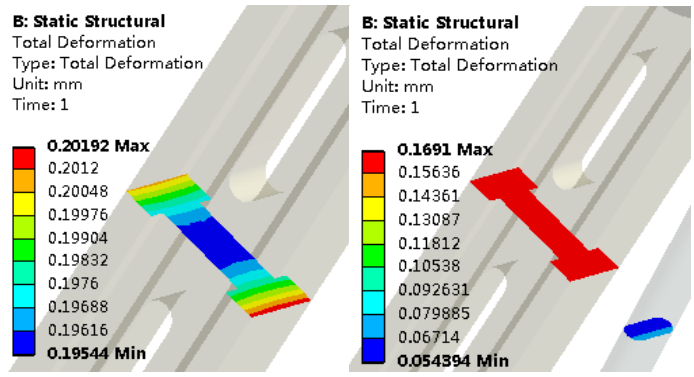

(b) Deformation distribution of foot

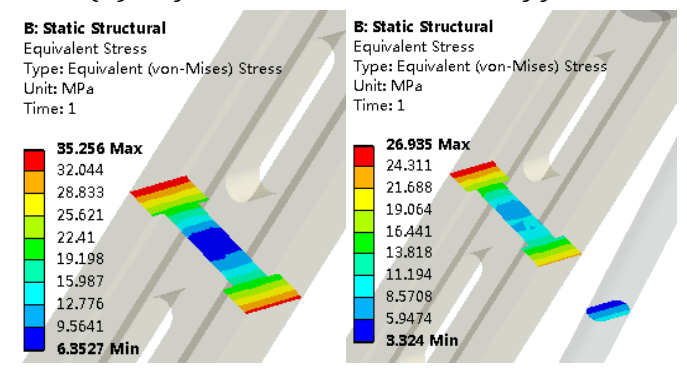

(c) Equivalent stress distribution of foot

Figure 14: Strength analysis with and without the tendon

Fig. 14(a) shows the strength analysis with and without the tendon. The principal stress is used to describe the actual stress of the robot leg. As can be seen from Fig. 14(a), the elastic tendon absorbs a portion of the ground reaction force in the actual stress after adding an elastic tendon, which conforms to the theoretical analysis. Thereby the impact of foot landing is eased and the force situation of the foot is improved.

Fig. 14(b) shows the stress distribution of foot with and without elastic tendon. The maximum foot deformation without elastic tendon in cross section is about $0.2019 \mathrm{~mm}$, and maximum foot deformation with elastic tendon in cross section is about $0.1694 \mathrm{~mm}$, total deformation decreased about $16.1 \%$.

Fig. 14(c) shows the equivalent stress distribution of foot with and without elastic tendon. The maximum equivalent stress of foot without elastic tendon is about $35.256 \mathrm{MPa}$, and the maximum equivalent stress of foot with elastic tendon is about $26.935 \mathrm{MPa}$, the maximum equivalent stress decreased about $23.6 \%$.

The above analysis can explain that the elastic tendon design is very beneficial, which eases the ground impact on the legs, improves the leg stress situation and prevents foot fracture.
Through several steps of locomotion trajectory optimization, lighten treatment and installation of elastic tendon, the high-speed test bench of locomotion robot leg design is completed, the pictures of finished prototype is shown in Fig. 15.

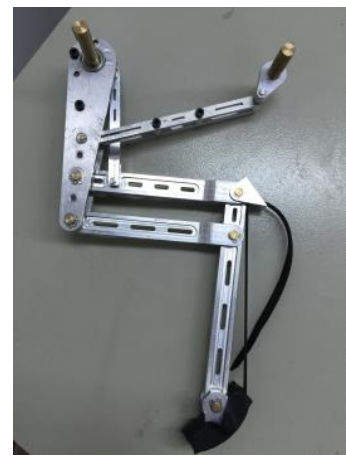

Figure 15: Prototype of mechanical leg

\section{Prototype Test}

In order to verify the rationality of the robot leg design, we conducted experiment in the selfdeveloped test bench of one-legged movement. Test platform is as shown in Fig.16:

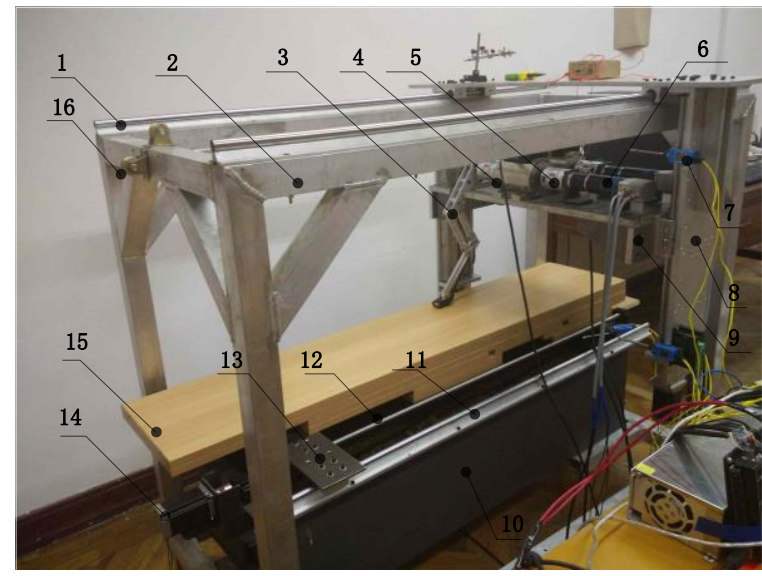

Figure 16: Diagram of test platform structures Description: 1. Rail; 2. Frame 1; 3. Robot leg; 4. Torque speed sensor; 5. Coupler; 6. Torque motor; 7. Stroke switch 2; 8. Screw slide module; 9. Work platform surface; 10. Earth tank; 11. Rail 2; 12. Ball screw; 13. Scraper; 14. Stepping motor; 15. Horizontal board; 16. Fixed pulley

The test platform drives work platform locomotion by a horizontal stepper motor and two vertical direction stepper motors, the work platform is equipped with the double four-bar robot leg test prototype proposed in this paper, and 2 speed torque sensors are equipped, the bottom soil tank of the test platform is equipped with paddy soils, horizontal boards can be placed on top of the soil tank, enabling one-legged robotic walking tests on paddy soils and hard pavements. 
I. Conduct walking test by setting the step distance as $300 \mathrm{~mm}$ and step height as $70 \mathrm{~mm}$, with zero impact foot trajectory, and constantly decrease gait cycle to verify the maximum speed the robot can reach. Through several tests, we found that the minimum gait cycle the robot can walk steadily is $0.6 \mathrm{~s}$, at this time, the walking process of the robot with an interval of $0.06 \mathrm{~s}$ is shown in Fig. 17, the test prototype is smooth in the walking process, and the gait is continuous and stable.
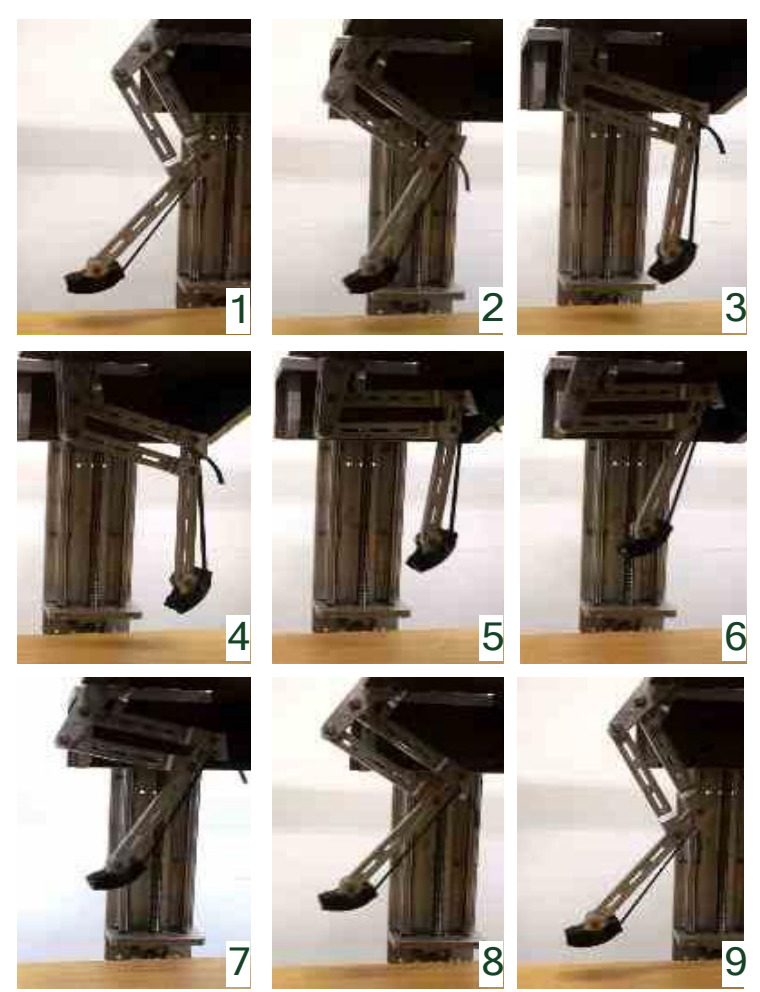

Figure 17: Experiment of robot at no-load walking

II. To test the correctness of the elastic tendon design, a smooth board is added above the soil tank to test the speed and torque of the hip and knee motors when the robot leg is walking on the board with the maximum speed, Mitsubishi PLC is adopted for data acquisition, and data is transmitted to PC host computer through the Modbus RTU protocol, which are shown in Fig. 18.

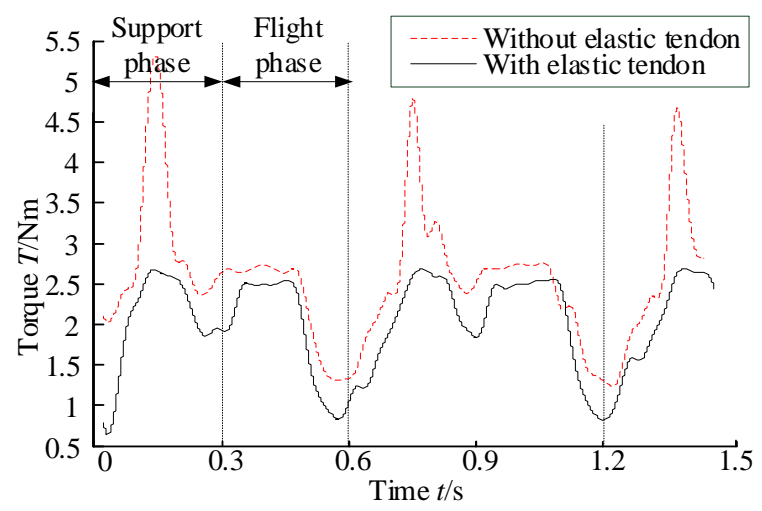

(a) Torque consumption of knee motor

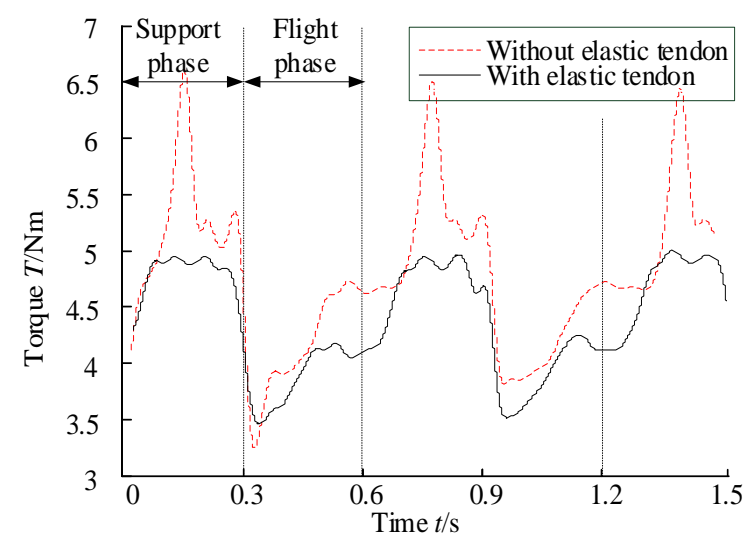

(b) Torque consumption of elbow motor

Figure 18: Torque consumption with and without the tendon

As shown in Fig. 18, the motor torque will have a large peak at the beginning of each locomotion cycle when there is no elastic tendon, which is consistent with the moment when leg just begins to land, that is to say the foot end of robot leg will receive a great impact when landing, the torque of two motors on the leg will also have great mutation; after the installation of elastic tendon, the torque curve has a stable change, there is no obvious fluctuations when the leg lands on ground, which can be considered that the elastic tendon wil absorb part of the impact at each landing moment, thus easing the stress of the leg, the torque of the two motors will not have very large mutation, which can reduce the requirements of the motor peak torque during high-speed locomotion, provide possibility for the selection of low-power and light motors, and verify the effectiveness of elastic tendon design.

\section{Conclusions}

(1) This paper introduces an novel electric-drive robot leg based on double four-bar mechanism, which is used to optimize the crank trajectory.

The final optimized foot trajectory is obtained by inserting a segment of constant speed locomotion and using linear interpolation fitting, the peak angular velocity of crank locomotion decreased $18.9 \%$, and the angular acceleration is also improved.

(2) On the basis of guaranteeing leg strength, lighten treatment is conducted on foot, and the mass reduced $23.4 \%$.

(3) Elastic tendon design is conducted on the legs, leg stress is improved by using the elastic tendon to absorb part of the ground impact force.

(4) In the test bench of one-legged movement test system of robot, the maximum locomotion speed of the robot leg is $1 \mathrm{~m} / \mathrm{s}$ ( $>2$ times of leg length). After adding an elastic tendon, the peak torque of the two motors decreased significantly. 


\section{Acknowledgements}

This research was supported by the National Natural Science Foundation of China (51405239) and the Fundamental Research Funds for the Central Universities (KY2201761).

\section{References}

[1] Hyun D J, Seok S, Lee J, et al. High speed trotrunning: Implementation of a hierarchical controller using proprioceptive impedance control on the MIT Cheetah[J]. The International Journal of Robotics Research. 2014, 33(11): 1417-1445.

[2] Maes L D, Herbin M, Hackert R, et al. Steady locomotion in dogs: temporal and associated spatial coordination patterns and the effect of speed[J]. Journal of Experimental Biology. 2008, 211(1): 138-149.

[3] Ananthanarayanan A, Azadi M, Kim S. Towards a bio-inspired leg design for high-speed running[J]. Bioinspiration \& biomimetics. 2012, 7(4): 4-12.

[4] Walter R M, Carrier D R. Ground forces applied by galloping dogs[J]. Journal of Experimental Biology. 2007, 210(2): 208-216.

[5] LIU Jing, ZHAO Xiaoguang, TAN Min. Legged robots: a review[J]. Robot. 2006, 28(1): 81-88.

[6] Seok S, Wang A, Chuah M Y, et al. Design principles for highly efficient quadrupeds and implementation on the MIT cheetah robot[C].2013 IEEE International Conference on Robotics and Automation (ICRA). IEEE, 2013: 3307-3312.

[7] Farve N N. Design of a low-mass high-torque brushless motor for application in quadruped robotics[D]. Massachusetts Institute of Technology, 2012.

[8] Estremera J, Waldron K J. Thrust control, stabilization and energetics of a quadruped running robot[J]. The International Journal of Robotics Research. 2008, 27(10): 1135-1151.

[9] Spröwitz A, Tuleu A, Vespignani M, et al. Towards dynamic trot gait locomotion: Design, control, and experiments with Cheetah-cub, a compliant quadruped robot[J]. The International Journal of Robotics Research. 2013, 32(8): 932-950.

[10] Hutter M, Gehring C, Bloesch M, et al. StarlETH: A compliant quadrupedal robot for fast, efficient, and versatile locomotion[C].15th International Conference on Climbing and Walking Robot-
CLAWAR 2012. 2012.

[11] Lewis M A, Bunting M R, Salemi B, et al. Toward ultra high speed locomotors: design and test of a cheetah robot hind $\operatorname{limb}[\mathrm{C}]$.Robotics and Automation (ICRA), 2011 IEEE International Conference on. IEEE, 2011: 1990-1996.

[12] Raibert M, Blankespoor K, Nelson G, et al. Bigdog, the rough-terrain quadruped robot[C].Proceedings of the 17th World Congress. Proceedings Seoul, Korea, 2008: 10822-10825.

[13] Sapaty P. Military Robotics: Latest Trends and Spatial Grasp Solutions[J]. International Journal of Advanced Research in Artificial Intelligence. 2015, 4(4): 9-18.

[14] CHAI Hui, MENG Jian, RONG Xuewen, et al. Design and implementation of SCalf, an advanced hydraulic quadruped robot[J]. Robot. 2014, 36(4): 385-391.

[15] Blickhan R, Seyfarth A, Geyer $H$, et al. Intelligence by mechanics[J]. Philosophical Transactions of the Royal Society of London A: Mathematical, Physical and Engineering Sciences. 2007, 365(1850): 199-220.

[16] Fischer M S, Blickhan R. The tri-segmented limbs of therian mammals: kinematics, dynamics, and self-stabilization-a review[J]. Journal of Experimental Zoology Part A: Comparative Experimental Biology. 2006, 305(11): 935-952.

[17] WANG Lipeng, WANG Junzheng, WANG Shoukun, et al. Strategy of foot trajectory generation for hydraulic quadruped robots gait planning[J]. Journal of Mechanical Engineering. 2013, 49(1): 39-44.

[18] Nie H, Sun R, Guo C, et al. Innovative Design and performance evaluation of a high-speed bionic mechanical leg[J]. Journal of Bionic Engineering. 2015, 12(3): 352-360.

[19] Yang Shu, Guo Dongming, Mi Dahai. Topology optimization of 3-D flexible micro-positioning design [J]. Transactions of the Chinese Society for Agricultural Machinery. 2008, 39(3): 131133.

[20] Rudman K E, Aspden R M, Meakin J R. Compression or tension? The stress distribution in the proximal femur[J]. Biomedical engineering online. 2006, 5(1): 1-7.

[21] Carter D R, Hayes W C. Bone compressive strength: the influence of density and strain rate[J]. Science. 1976, 194(1): 174-1176. 